\title{
A promoter polymorphism of the insulin-like growth factor-I gene is associated with left ventricular hypertrophy
}

\author{
G S Bleumink, A F C Schut, M C J M Sturkenboom, J A M J L Janssen, J C M Witteman, C M van \\ Duijn, A Hofman, B H Ch Stricker
}

carton eft ventricular hypertrophy ( $\mathrm{LVH})$ is a strong predictor of cardiovascular morbidity and mortality. Insulin-like growth factor-I (IGF-I) has an important role in the hypertrophic response of the myocardium. ${ }^{1}$ A polymorphism in the IGF-I gene promoter region has been identified which influences IGF-I production. ${ }^{2-4}$ Studying this polymorphism in relation to pathology may better reflect the effects of long term IGF-I exposure than studies on serum IGF-I concentrations, which may fluctuate considerably and are profoundly influenced by various factors. We investigated the association between this IGF-I promoter polymorphism and the occurrence of LVH on echocardiogram.

\section{METHODS}

The Rotterdam study is a population based cohort study in 7983 elderly people aged 55 years or over. The baseline examination was conducted in 1990-93, during which time information was obtained on age, sex, history of myocardial infarction, smoking, hypertension, diabetes mellitus, and body mass index (BMI). Cardiac ultrasound was performed in a random subpopulation of 2823 subjects. The present study was performed in subjects aged $55-75$ years, without a history of myocardial infarction. The analyses were restricted to persons with an adequate echocardiogram and for whom blood specimens were available for IGF-I typing, a total of 1678 subjects. This is the first genetic case association study that uses the Rotterdam study database to investigate the phenotype of LVH on the echocardiogram.

$M$ mode echocardiography was performed by experienced staff, according to recommendations of the American Society of Echocardiography (ASE). Left ventricular mass was determined using the Devereux modified ASE cube formula: left ventricular mass $(\mathrm{g})=0.8 \times(1.04 \times[(\mathrm{LVED}+\mathrm{IVS}+$ LVPW $\left.\left.)^{3}-(\text { LVED })^{3}\right]\right)+0.6$, where LVED is the left ventricular end diastolic diameter, IVS is the interventricular septum thickness, and LVPW is the left ventricular posterior wall thickness. Left ventricular mass was indexed to body surface area. Using predefined criteria, based on use in recent clinical trials and ability to predict cardiovascular prognosis, LVH was defined as having a left ventricular mass index $\geqslant 104 \mathrm{~g} / \mathrm{m}^{2}$ in women and $\geqslant 116 \mathrm{~g} / \mathrm{m}^{2}$ in men. ${ }^{56}$ As consensus concerning LVH thresholds is lacking, left ventricular mass index was also divided into sex specific quintiles.

The polymorphism under study was a cytosine-adenosine repeat in the promoter region of the IGF-I gene. Genotyping was performed as described elsewhere. ${ }^{3}$ Earlier, we identified 10 different alleles at this single locus in the IGF-I gene in a sample of 900 subjects of the Rotterdam study. ${ }^{3}$ Of these participants, $88.4 \%$ carried at least one 192 base pair allele, corresponding to 19 cytosine-adenosine repeats, suggesting that this is the wild type allele. The frequency of the other nine alleles was low. Based on this observation, using the wild type allele, our study population was divided into three genotypes: individuals homozygous for the 192 base pair allele (43.6\%), individuals heterozygous for the 192 base pair allele $(45.6 \%)$, and non-carriers of the 192 base pair allele (10.7\%).

Hardy-Weinberg equilibrium was tested using a $\chi^{2}$ test. We used multivariate logistic regression to calculate odds ratios (OR) plus 95\% confidence intervals (CI) for the association between LVH and IGF-I genotypes. An allele dose effect model was assumed to explain differences between genotype groups. Participants homozygous for the 192 base pair allele served as the reference category. Allele dose-effect relation was tested with age and sex adjusted logistic regression (trend test). Men and women were pooled in the analyses because there was no effect modification by sex.

\section{RESULTS}

We identified 358 cases with LVH. Genotype and allele distributions were in Hardy-Weinberg equilibrium. Participants with LVH were on average two years older (mean age 65 versus 63 years, $t$ test $\mathrm{p}<0.001$ ), had a higher BMI, and had diabetes mellitus and hypertension more often. Sex and smoking did not differ significantly between participants with or without LVH.

Table 1 shows that non-carriers of the 192 base pair allele had a 1.5 fold increased risk of LVH compared to individuals homozygous for the 192 base pair allele (adjusted OR 1.49, $95 \%$ CI 1.01 to 2.20). Although the frequency of LVH was not significantly increased in heterozygotes, there was a significant allele dose-effect relation. The odds of LVH were multiplied by 1.2 per non-wild type allele present. Findings were similar for the association between IGF-I genotypes and the highest sex specific quintile versus other sex specific quintiles of left ventricular mass index (age adjusted OR noncarriers $1.48,95 \%$ CI 1.001 to 2.18 ). In multivariate analysis this association was, however, not significant, although the OR remained approximately the same (OR 1.46, 95\% CI 0.99 to 2.17 ).

\section{DISCUSSION}

In this study, non-carriers of the 192 base pair allele of a cytosine-adenosine repeat in the promoter region of the IGF-I gene were more susceptible to the development of LVH than participants homozygous for the wild type allele. In addition, there was a significant allele dose-effect relation.

Several studies have investigated the potential functionality of the IGF-I polymorphism with conflicting results. The IGF-I polymorphism has been associated with serum IGF-I concentrations, albeit with opposite directions. ${ }^{2-4} 7$ Differences in population background (environmental and genetic factors), age, and small sample sizes may all account for these contradictory results. However, findings in Dutch populations have been very consistent, strongly suggesting

Abbreviations: ASE, American Society of Echocardiography; BMI, body mass index; IGF-I, insulin-like growth factor-I; LVH, left ventricular hypertrophy 
Table 1 Association between echocardiographically determined LVH and IGF-I genotypes

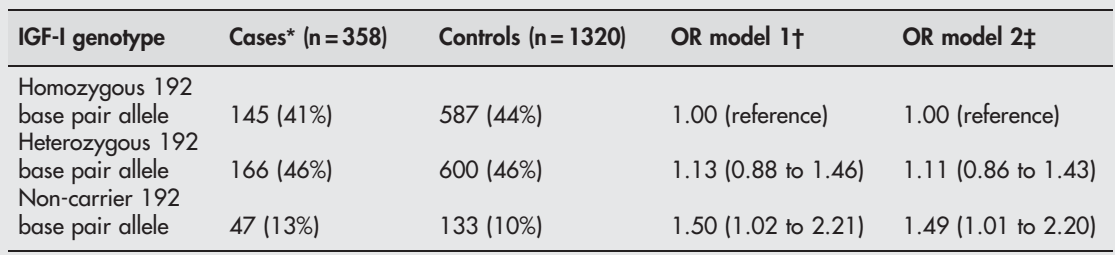

* $\mathrm{LVH}$ was considered present when left ventricular mass index was $\geqslant 104 \mathrm{~g} / \mathrm{m}^{2}$ in women and $116 \mathrm{~g} / \mathrm{m}^{2}$ in men. †Adjusted for age and sex, allele effect relation $p$ trend $<0.05$

$\ddagger$ Adjusted for age, sex, BMI, hypertension, and diabetes mellitus

Data are presented as percentages of cases or controls and as odds ratios (OR) with $95 \%$ confidence interval.

that the IGF-I polymorphism can be used as a genetic marker, at least in Dutch subjects. ${ }^{38}$ Recently, we found that non-carriers of the 192 base pair allele had lower circulating total IGF-I concentrations and lower body height than carriers. The absence of this allele was significantly associated with an increased risk of type 2 diabetes mellitus and myocardial infarction. ${ }^{3}$ Moreover, we observed that the normal gradual decline in circulating serum IGF-I concentrations during aging was highly influenced by this polymorphism in the IGF-I gene. ${ }^{4}$ Evidence suggests that IGF-I deficiency may lead to diminished cardiac performance and adverse remodelling in reaction to an injury to the heart. ${ }^{1}$

Confounding bias or chance may provide alternative explanations for our results. Confounding bias is unlikely, as we studied a homogeneous white population and potential confounding factors did not greatly influence our results. Chance cannot be fully excluded, since the probability that we incorrectly rejected our null hypothesis of no association was less than $5 \%$.

In conclusion, non-carriers of a 192 base pair allele polymorphism in the promoter region of the IGF-I gene are more susceptible to the development of LVH than participants homozygous for the wild type allele. This may result from a relative IGF-I deficiency, leading to faulty remodelling in response to myocardial injury.

\section{Authors' affiliations}

G S Bleumink; A F C Schutt, M C J M Sturkenboom, J C M Witteman, C M van Duijn, A Hofman, B H Ch Stricker*, Department of Epidemiology \& Biostatistics, Erasmus MC, Rotterdam, The Netherlands J A M J L Janssen, Department of Internal Medicine, Erasmus MC
*Also at Inspectorate for Healthcare, The Hague, The Netherlands; talso at Department of Internal Medicine, Erasmus MC

Correspondence to: Dr Bruno H Ch Stricker, Pharmaco-epidemiology Unit, Department of Epidemiology \& Biostatistics, Erasmus MC, PO Box 1738, 3000 DR Rotterdam, The Netherlands; b.stricker@erasmusmc.nl

Accepted 15 April 2004

\section{REFERENCES}

1 Ren J, Samson WK, Sowers JR. Insulin-like growth factor I as a cardiac hormone: physiological and pathophysiological implications in heart disease. J Mol Cell Cardiol 1999;31:2049-61.

2 Rosen CJ, Kurland ES, Vereault D, et al. Association between serum insulin growth factor-I (IGF-I) and a simple sequence repeat in IGF-I gene: implications for genetic studies of bone mineral density. J Clin Endocrinol Metab 1998;83:2286-90.

3 Vaessen N, Heutink P, Janssen JA, et al. A polymorphism in the gene for IGF-I. Functional properties and risk for type 2 diabetes and myocardial infarction. Diabetes, $2001 ; 50: 637-42$.

4 Rietveld I, Janssen JAMIL, Hofman A, et al. A polymorphism in the IGF-I gene influences the age-related decline in circulating total IGF-I levels. Eur J Endocrinol 2003;148:171-5.

5 Palmieri V, Dahlöf B, DeQuattro V, et al. Reliability of echocardiographic assessment of left ventricular structure and function. The PRESERVE study. J Am Coll Cardiol 1999;34:1625-32.

6 Wachtell K, Bella JN, Liebson PR, et al. Impact of different partition values on prevalences of left ventricular hypertrophy and concentric geometry in a large hypertensive population. The LIFE study. Hypertension 2000;35:6-12.

7 Frayling TM, Hattersley AT, McCarthy A, et al. a putative functional polymorphism in the IGF-I gene. Association studies with type 2 diabetes, adult height, glucose tolerance, and fetal growth in U.K. populations. Diabetes 2002;51:2313-6.

8 Arends N, Johnston L, Hokken-Koelenga A, et al. Polymorphism in the IGF-I gene: clinical relevance for short children born small for gestational age (SGA). J Clin Endocrinol Metab 2002;87:2720-4. 